\title{
ANÁLISE DA ASSISTÊNCIA PRESTADA À PESSOA COM SURDEZ NA PERCEPÇÃO DOS PROFISSIONAIS DA SAÚDE NA APS DOS MUNICÍPIOS DE PARNAÍBA - PI E LUÍS CORREIA - PI
}

\author{
ANALYSIS OF THE ASSISTANCE PROVIDED TO DEAF PEOPLE IN THE PERCEPTION OF HEALTH PROFESSIONALS IN PRIMARY \\ HEALTH CARE IN THE MUNICIPALITIES OF PARNAÍBA - PI AND LUÍS CORREIA - PI
}

BARROS, Esther Barata Machadoํㅗ NASCIMENTO, VASCONCELOS, Any Carolina Cardoso Guimarães¹, Clesivane do Socorro Silva do 1

${ }^{1}$ Faculdade de Ciências Humanas, Exatas e da Saúde do Piauí/Instituto de Educação Superior do Vale do Parnaíba (FAHESP/IESVAP).

\begin{abstract}
RESUMO
A Língua Brasileira de Sinais (LIBRAS), reconhecida pela Lei Federal oㅜ 10.436/2002 e regulamentada pelo Decreto no 5.626/2005, é um meio legal de comunicação e expressão utilizado pela comunidade surda no Brasil. O conhecimento e utilização dessa modalidade comunicativa pelos profissionais nos ambientes de saúde é indispensável para a escuta qualificada, compreensão mútua e atenção integral do paciente surdo, o que envolve desde a porta de entrada do sistema até os níveis mais complexos da atenção. Este trabalho tem como objetivo analisar o atendimento oferecido à pessoa com surdez e as dificuldades de estabelecer a comunicação com esse grupo na Atenção Primária à Saúde (APS). Trata-se de um estudo descritivo, com abordagem qualitativa e quantitativa, direcionado aos profissionais da APS dos municípios de Parnaíba e Luís Correia - PI. A pesquisa foi realizada entre os meses de novembro de 2018 e fevereiro de 2019 e os dados coletados a partir de um questionário adaptado semiestruturado de autopreenchimento, avaliados pelo método Análise de Conteúdo de Bardin. Foram definidas duas categorias temáticas: Comunicação entre o profissional de saúde e o usuário surdo e Atenção à saúde da pessoa com surdez na APS. Após análise dos resultados, infere-se que dos 32 participantes da pesquisa, $50 \%$ já haviam abordado pacientes com surdez em seus atendimentos, $78 \%$ não sabiam informar a quantidade de pessoas surdas na sua área de atuação e cerca de 47\% afirmaram nunca ter realizado estratégias para incluir este grupo nas Unidades Básicas de Saúde (UBS) onde trabalham. Portanto, o conhecimento da língua de sinais nos locais de atenção à saúde consolida o processo de cuidado e o acesso integral da pessoa com surdez aos serviços de saúde e, sobretudo, a superação da barreira comunicativa que tanto afasta os profissionais do paciente com surdez.
\end{abstract}

Palavras-chave: Surdez. Atenção Primária. Barreiras de Comunicação.

\begin{abstract}
The Brazilian Sign Language (LIBRAS), recognized by Federal Law No. 10,436 / 2002 and regulated by Decree No. 5,626 / 2005, is a legal means of communication and expression used by the deaf community in Brazil. The knowledge and use of this communicative modality by professionals in health environments is indispensable for qualified listening, mutual understanding, and comprehensive care of the deaf patient, which involves from the system's entrance door to the most complex levels of care. This work has as objective analyze the care offered to deaf people and the difficulties to establish communication with this group in Primary Health Care (PHC). This is a descriptive study, with qualitative and quantitative approaches, directed to PCH's professionals in the cities of Parnaíba and Luis Correia - PI. The research was carried out between the months of November 2018 and February 2019, and the data were collected through a self-filling semi-structured adapted questionnaire, evaluated by Bardin's Content Analysis method. Two themed categories were defined: Communication between the health professional and the deaf patient, and the care of deaf people's health in PHC. After analyzing the results, it appears that of the 32 survey participants, 50\% had already approached deaf patients in their care, $78 \%$ could not inform the amount of deaf people in their area of operation and about $47 \%$ said they had never had any strategies to include this group in the Basic Health Units (BHU) where they work. Therefore, knowledge of sign language in health care places consolidates the care process and the deaf person's full access to health services and, above all, the overcoming of the communicative barrier that separates professionals from the deaf patient.
\end{abstract}

Keywords: Deafness. Primary Care. Communication Barriers. 


\section{INTRODUÇÃO}

A Língua Brasileira de Sinais (LIBRAS), reconhecida pela Lei Federal no 10.436/2002 e regulamentada pelo Decreto no 5.626/2005, é considerada como meio legal de comunicação e expressão utilizado pela comunidade surda no Brasil ${ }^{1}, 2$. A LIBRAS, assim como outras línguas, apresenta estruturas gramaticais próprias e peculiaridades, tais como a criatividade, a produtividade ilimitada, a multiplicidade de funções e a articulação dos elementos no plano do conteúdo e da expressão, que a torna uma língua natural, legítima e a distingue das outras modalidades existentes ${ }^{3}$.

De acordo com o Censo Demográfico realizado em 2010 pelo Instituto Brasileiro de Geografia e Estatística (IBGE), existem cerca de 46 milhões de pessoas com algum tipo de deficiência no Brasil, sendo que 1,1\% delas apresentam deficiência auditiva ${ }^{4}$. Estes dados demonstram a necessidade em compreender e utilizar a LIBRAS em todos os setores da sociedade, sobretudo nos serviços de saúde oferecidos.

O Sistema Único de Saúde (SUS) apresenta como princípios doutrinários a Universalidade, Equidade e a Integralidade e pode ser compreendido como uma rede de atendimento articulada a pontos de atenção à saúde 5 . Nesse aspecto, estratégias foram elaboradas para oferecer apoio para a população surda no Brasil, como a Política Nacional de Saúde da Pessoa Portadora de Deficiência (PNSPPD).

Essa política é uma estratégia voltada para a inclusão das pessoas com deficiência em todas as esferas da vida social. De acordo essa estratégia, as ações de assistência à saúde direcionadas para esse grupo na APS devem estar incluídas em um sistema amplo, que envolva simultaneamente o indivíduo, sua família e a comunidade no processo de reabilitação e cuidado ${ }^{6}$. Desse modo, a Atenção Primária à Saúde é a porta de entrada do sistema e funciona como um local fundamental para a organização do cuidado à saúde dos indivíduos, suas famílias e da comunidade ${ }^{7}$.

A Política Nacional de Atenção à Saúde Auditiva (PNASA) instituída pelo Ministério da Saúde em 2004, institui que a atenção básica deve realizar ações de caráter individual ou coletivo, direcionadas para a promoção da saúde das pessoas com surdez, prevenção e identificação de problemas auditivos e desenvolvimento de estratégias informativas, educativas e de orientação familiar ${ }^{8}$.

Diante disso, o papel exercido pelas equipes de saúde da atenção primária na integralidade do cuidado aos usuários e o domínio da LIBRAS por esses profissionais são essenciais para o acolhimento da pessoa com surdez, pois a procura deste grupo por atendimento nos serviços de saúde é menos frequente e de forma mais tardia, quando comparado a pessoa ouvinte ${ }^{9}$.

O conhecimento e utilização da língua de sinais pelos profissionais nos ambientes de saúde é indispensável para a escuta qualificada, compreensão mútua e atenção integral do paciente surdo, o que envolve desde a porta de entrada do sistema (atenção primária) até os níveis mais complexos da atenção (atenção secundária e terciária). O processo de acolhimento e a construção da relação médico e/ou profissional ouvinte e o paciente surdo deve ser alcançada sem intermediários, mediante estratégias inclusivas e humanizadas que ultrapassem as barreiras comunicativas e o distanciamento, historicamente existente, entre esses dois grupos. Portanto, o domínio da Língua Brasileira de Sinais é uma das principais ferramentas para consolidar o processo de cuidado e atenção à saúde da pessoa com surdez.

\section{MÉTODOS}

Trata-se de um estudo descritivo com abordagem qualitativa e quantitativa, direcionado aos profissionais da Atenção Primária à Saúde dos municípios de Parnaíba - PI e Luís Correia - PI.

Os dados foram coletados no período de 3 meses, entre novembro de 2018 e fevereiro de 2019, após aprovação pelo Comitê de Ética em Pesquisa (CEP) do Centro Universitário Tocantinense Presidente Antônio Carlos UNITPAC, parecer oㅜ 3.009.943, de 08/11/18. Mediante à Carta de Anuência às Secretarias de Saúde dos municípios de Parnaíba - PI e Luís Correia - PI foi solicitada e autorizada a realização do estudo nas Unidades Básicas de Saúde (UBS) escolhidas.

A amostra do estudo foi composta por 32 profissionais atuantes nas unidades básicas de saúde Alto Santa Maria, Mendonça Clark e Rodoviária localizadas no município de Parnaíba e na UBS Alto Bonito no município de Luís Correia, que aceitaram participar da pesquisa, mediante à assinatura do Termo de Consentimento Livre e Esclarecido (TCLE).

Os critérios de inclusão da pesquisa foram os profissionais da saúde em exercício nas UBS selecionadas para participar da pesquisa, que tinham ou não atendido pessoas com surdez, com ou sem o conhecimento da LIBRAS e que concordassem em participar da pesquisa, mediante a assinatura do TCLE. Foram excluídos os profissionais da saúde que não trabalhavam nas UBS selecionadas, aqueles que não concordaram em participar do estudo e os que não assinaram o TCLE.

Como instrumento de coleta dos dados foi utilizado um questionário adaptado semiestruturado (Anexo A) de autopreenchimento, contendo 10 perguntas fechadas, 9 perguntas abertas e 3 perguntas mistas, com questões relacionadas ao perfil demográfico dos entrevistados, a comunicação com a população surda e os aspectos referentes à organização dos serviços de saúde ${ }^{10}$. Os questionários foram entregues juntos com o TCLE em envelopes fechados para todos os profissionais que desejaram participar. Foi estabelecido um prazo de 7 dias entre a pesquisadora e os participantes para o recolhimento do questionário. A participação dos entrevistados foi voluntária, sem a cobrança de qualquer quantia ou geração de gastos decorrentes da participação.

A análise dos dados da pesquisa foi fundamentada no método de Análise de Conteúdo de Bardin, o qual possibilita a sistematização progressiva do conhecimento para a melhor compreensão do objeto de estudo e o desenvolvimento de novos conceitos acerca dos elementos investigados ${ }^{11}$. Com o objetivo de analisar os relatos dos participantes da pesquisa e garantir o sigilo e a confidencialidade, foi utilizada a letra inicial da palavra 
profissional $(P)$, seguida de um numeral conforme a ordem de análise dos dados (P1, P2, P3 etc.).

\section{RESULTADOS E DISCUSSÃO}

Dentre os 32 participantes da pesquisa foi observado uma predominância do gênero feminino $(81,25 \%)$ em relação ao masculino $(18,75 \%)$, com média de idade entre 39,5 anos e 36,6 anos, respectivamente. Os dados da Tabela 1 revelam que os participantes do estudo apresentam em média 12 anos de formação profissional e cerca 8 anos de atuação nas UBS em que se realizou a pesquisa. Dentre os participantes foi observado, quanto ao cargo que desempenhavam nas unidades, um total de 3 médicos, 13 Agentes Comunitários de Saúde, 3 Enfermeiros, 4 Técnicos de Enfermagem, 2 Técnicos de Saúde Bucal, 4 Recepcionistas e 3 Prestadores de Serviços Gerais.

Tabela 1 Caracterização profissional dos participantes.

\begin{tabular}{lcc}
\hline Características & $\mathbf{n}$ & $\%$ \\
\hline Tempo como profissional (Anos) & & \\
Média & 12,18 & ---- \\
Tempo na UBS (Anos) & & \\
Média & 8,13 & ---- \\
Setor em que trabalha & & \\
Médico (a) & 3 & $9,4 \%$ \\
ACS & 13 & $40,6 \%$ \\
Enfermeiro (a) & 3 & $9,4 \%$ \\
Téc. de Enfermagem (a) & 4 & $12,5 \%$ \\
Téc. de Saúde Bucal (a) & 2 & $6,2 \%$ \\
Recepcionista & 4 & $12,5 \%$ \\
Serviços Gerais (a) & 3 & $9,4 \%$ \\
Total & 32 & $100,0 \%$ \\
\hline
\end{tabular}

Fonte: Dados da pesquisa.

Conforme exemplificado na Tabela 2, a capacitação em Língua Brasileira de Sinais foi realizada por cerca de $28 \%$ dos participantes e $68,8 \%$ afirmam não ter feito curso de LIBRAS, apesar de saber da existência da língua. Dentre os 9 participantes que tiveram formação nessa língua, $66,6 \%$ fizeram o curso em uma disciplina da faculdade, $11,2 \%$ em escolas de ensino de línguas e $22,2 \%$ em outros locais.

A Tabela 3 explicita questões relacionadas ao conhecimento do número de pacientes surdos adscritos na área e se os participantes já haviam atendido algum paciente surdo. Os dados relevam que dos 32 entrevistados, $78,1 \%$ não sabiam informar a quantidade de pessoas com surdez na sua área de atuação e $50 \%$ já haviam abordado pessoas surdas em seus atendimentos.

De acordo com os dados coletados na pesquisa foram estabelecidas duas categorias temáticas: Categoria 1: Comunicação entre o profissional de saúde e o usuário surdo na APS, com a Subcategoria 1.1: Ausência e/ou presença de intérprete durante 0 atendimento; e Categoria 2: Atenção à saúde da pessoa com surdez na APS, com a Subcategoria 2.1: Manejo da equipe.

Tabela 2 Capacitação em Língua Brasileira de Sinais (LIBRAS).

\begin{tabular}{lrr}
\hline & $\mathbf{n}$ & $\%$ \\
\hline Já fez algum curso de LIBRAS? & & \\
Sim & 9 & $28,1 \%$ \\
Não, mas sei que essa língua existe & 22 & $68,8 \%$ \\
Não e não sei que língua é essa & 1 & $3,1 \%$ \\
Total & 32 & $100,0 \%$ \\
Local em que realizou o curso & & \\
Escola de ensino de línguas & 1 & $11,2 \%$ \\
Em uma disciplina na faculdade & 6 & $66,6 \%$ \\
Outros & 2 & $22,2 \%$ \\
Total & $9 *$ & $100 \%$ \\
\hline${ }^{*}$ Total de participantes que afirmaram ter feito curso de LIBRAS & & \\
\hline
\end{tabular}

Fonte: Dados da pesquisa.

Tabela 3 Pessoas com surdez nas UBS do estudo.

\begin{tabular}{lrr}
\hline & $\mathbf{n}$ & $\%$ \\
\hline Sabe quantos surdos estão adscritos na área? & & \\
Sim & 7 & $21,9 \%$ \\
Não & 25 & $78,1 \%$ \\
Total & 32 & $100,0 \%$ \\
Já atendeu algum paciente surdo? & & \\
Sim & 16 & $50 \%$ \\
Não & 16 & $50 \%$ \\
Total & 32 & $100 \%$ \\
\hline
\end{tabular}

Fonte: Dados da pesquisa.

\section{Categoria 1: Comunicação entre o profissional de saúde e o usuário surdo na APS}

"Não conheço muito a língua de sinais, porém faria o possível para que o paciente compreendesse o que eu queria passar. Até desenharia se possível." (P12)

A comunicação é uma ferramenta indispensável para a humanização das relações de cuidado, interação entre a equipe de saúde e o usuário e sua família, bem como para o compartilhamento de saberes ${ }^{12}$. As habilidades comunicativas são importantes ferramentas para a assistência de todos os usuários do sistema de saúde e dão suporte para as ações dos profissionais ${ }^{13}$.

Essa categoria foi avaliada no questionamento a respeito do atendimento ao paciente surdo na ausência de um intérprete ou familiar/conhecido acompanhante. Cerca de $75 \%$ profissionais da saúde afirmaram que atenderiam esse paciente sem um intermediário, apesar das dificuldades que pudessem surgir pela falta de 
compreensão entre ambas as partes, como pode ser analisado nos seguintes depoimentos:

"Buscaria formas de me comunicar com ele, o pouco de LIBRAS que sei colocaria em prática, o que não pode acontecer é deixar o paciente sem atendimento por uma dificuldade que é minha e não dele." (P3)

"Como já tive a oportunidade de pagar uma disciplina de LIBRAS e também fiz um curso básico, se tiver uma conversa dá pra entender um pouco." (P5)

"Tentaria me comunicar da melhor forma possível." (P9)

O processo comunicativo deve envolver dimensões verbais e não verbais (postura, gestos e expressão facial) e garantir a clareza na transmissão da mensagem. A comunicação deve oferecer autonomia ao usuário/cuidador para a tomada de decisões em relação a terapêutica proposta e as condições necessárias para se estabelecer o autocuidado $^{12}$.

Os problemas de comunicação interpessoal existem em todo sistema de saúde e tornam-se mais expressivos quando estão relacionadas às barreiras de linguagem. Os problemas comunicativos associados ao distanciamento entre o paciente surdo e o profissional da saúde ainda são persistentes. Na maior parte dos casos, esse quadro está relacionado ao desconhecimento, insegurança e/ou a falta de habilidades em utilizar a língua de sinais por aqueles que prestam assistência na área da saúde ${ }^{14}$.

"Tenho a convicção que não conseguiria compreendê-lo." (P24)

"Minha comunicação com ele seria impossível, nós não nos entenderíamos." (P22)

"Com bastante dificuldade, mas tentaria escrever ou utilizar números." (P25)

Apesar da atenção básica ser a porta de entrada do SUS e ter o papel de realizar o acolhimento inicial desse usuário ${ }^{(15)}$, o que se observa na maior parte dos relatos é o referenciamento do paciente surdo para os níveis especializados de atenção à saúde e o seu distanciamento das profissionais das unidades de saúde locais.

"Encaminharia para o setor competente ou tentaria marcar outro encontro com o intérprete." (P25)

"Tentaria atender, caso não me sentisse segura encaminharia para o centro de especialidades." (P18)

"Encaminharia para outro profissional que tivesse condições de atendê-lo." (P22)

\section{Subcategoria 1.1: Ausência e/ou presença de intérprete durante 0 atendimento}

A pessoa com surdez, na maior parte das vezes, precisa de indivíduos que intermedeiem a relação com os profissionais da saúde e traduzam as emissões de ambas as partes durante 0 atendimento ${ }^{16}$. A presença de um intérprete da Língua Brasileira de Sinais ou um profissional capacitado para se comunicar nessa modalidade linguística nos ambientes de saúde é essencial ${ }^{17}$ e auxilia no processo comunicativo, sobretudo no que diz respeito a coleta de informações do paciente ${ }^{12}$.

"Melhor entendimento para quem está atendendo"

"Facilita entender o que o paciente está sentindo."

"Segurança de um diálogo mais claro e objetivo para que ambos se entendam." (P3)

A Lei Federal no 10.436/2002 regulamenta que o atendimento às pessoas com surdez na rede de serviços do SUS deve ser feito por profissionais devidamente capacitados para o uso da LIBRAS ou para sua tradução e interpretação. Conforme preceitua o Art. 3o, "as instituições públicas e empresas concessionárias de serviços públicos de assistência à saúde devem garantir atendimento e tratamento adequado aos portadores de deficiência auditiva, de acordo com as normas legais em vigor"1.

O que se observa na maior parte dos casos é a presença da figura familiar ou de pessoas próximas à pessoa surda funcionando como intermediadores do diálogo. Quando questionados sobre a presença do intérprete, familiar ou conhecido, $40,6 \%$ dos participantes afirmaram ser necessária durante 0 atendimento e que contribui para a comunicação, conforme observado nos relatos:

"Foi necessário a ajuda de um familiar, pois não consegui entender o que o usuário estava a informar." (P24)

"A presença do familiar é fundamental para haver “comunicação"'. (P27)

"Confiança, segurança que o usuário demonstra com o familiar." (P24)

Os dados da pesquisa revelam que grande parte dos acompanhantes dos pacientes surdos eram pessoas da família. Embora o familiar atue como facilitador do diálogo, essa intermediação pode acarretar prejuízos no processo comunicativo. A pessoa com surdez pode não transmitir suas informações de forma autêntica para os profissionais da saúde na presença do familiar ou conhecido ${ }^{13}$.

"Não traz autonomia para a pessoa com deficiência, o que pode ocasionar outros problemas como baixa autoestima" (P21)

"O diálogo fica bem limitado, dá a impressão que não traduziu tudo." (P28)

"Os pontos negativos vão desde a certeza que estão corretas as informações repassadas até a incapacidade de compreendê-la pessoalmente." (P24)

O intérprete deve interferir o mínimo possível na transmissão das informações e traduzir de forma fidedigna o conteúdo das mensagens ${ }^{18}$. O comprometimento da privacidade e do sigilo nos atendimentos é um problema que deve ser levado em consideração ${ }^{13}$. O paciente com 
surdez pode omitir informações imprescindíveis para a sua saúde e bem estar por vergonha, constrangimento ou desconfiança diante de uma terceira pessoa intermediando o diálogo com o profissional da saúde ${ }^{10}$.

"[...] dependendo do caso acredito que o paciente fica com receio de relatar fidedignamente alguma queixa diante de um intérprete." $(P 3)$

"A individualidade do atendimento, bem como a relação direta com o paciente ficam prejudicados." (P25)

"Em um dos casos senti a falta de fidelidade nas informações referidas" (P27)

\section{Categoria 2: Atenção à saúde da pessoa com surdez na APS}

Os participantes da pesquisa foram questionados sobre as estratégias utilizadas pelas equipes locais para a inclusão do paciente surdo nas ações de saúde desenvolvidas.

"Para falar a verdade, nunca pensamos em uma forma de incluir." (P19)

"[...] se os profissionais fossem capacitados para tal atividade não precisaria de um intérprete." (P10)

De acordo com a Tabela 4, cerca de 47\% dos entrevistados afirmaram, até o período em que foi realizada a pesquisa, que nunca realizaram estratégias para incluir as pessoas com surdez nas UBS onde trabalham e, somente $12,5 \%$ apontam que sempre incluem esse grupo nas ações de saúde.

As atividades desenvolvidas pelos profissionais da saúde devem envolver, além da competência técnica, os conhecimentos acerca da abordagem integral ao paciente $^{19}$. As estratégias devem levar em consideração a individualidade, as limitações e/ou deficiências de cada sujeito no processo de cuidado.

\section{Subcategoria 2.2: Manejo da equipe}

As ações e serviços realizados pelos profissionais entrevistados que já haviam atendido um paciente surdo concentraram-se em: pré-consultas, acompanhamento de hipertensão e diabetes, avaliação odontológica/ tratamento dentário e consultas ambulatoriais, sendo esta última a mais frequente.

Pode-se analisar que independente da atividade direcionada para a pessoa com surdez, o conhecimento da LIBRAS torna-se imprescindível. Quando questionados sobre as ações de inclusão do paciente surdo, a capacitação em língua de sinais foi a sugestão mais referida entre os participantes como a estratégia inicial para a abordagem a esse grupo.

"Capacitação dos funcionários, o principal ponto, sem isto fica complicado um atendimento de qualidade. $O$ resto seria consequência. Profissionais preparados saberiam melhor como os incluir." (P12)

"Os profissionais têm que primeiro se capacitar em libras para depois definir as melhores estratégias." (P25)

"[...] qualificar os profissionais de saúde para que tenham estratégias e conhecimentos para atuar e incluir esses pacientes no âmbito das ações e serviços de saúde da Atenção Básica.” (P30)
A III Conferência Nacional dos Direitos da Pessoa com Deficiência realizada em 2012 elencou a capacitação continuada dos profissionais em LIBRAS, Braille e outras metodologias de comunicação como uma das estratégias prioritárias para a abordagem mais adequada da pessoa com deficiência em todos os níveis de atenção à saúde ${ }^{20}$.

A capacitação em LIBRAS deve ser direcionada para todos os profissionais da área, de acordo com a demanda e a realidade local. A participação da pessoa com surdez torna-se ferramenta importante para enriquecer o aprendizado da língua de sinais $^{12}$, na medida em que favorece o conhecimento das particularidades linguísticas e culturais da comunidade surda ${ }^{19}$, aproxima os participantes às necessidades de saúde do paciente surdo, bem como auxilia na busca ativa desses usuários nas áreas adscritas $^{10}$.

\section{CONCLUSÃO}

A barreira comunicativa entre o paciente surdo e as equipes continua sendo o grande desafio nos ambientes de saúde. Embora existam leis e decretos que reconhecem a LIBRAS como a segunda língua oficial do Brasil e a língua materna da comunidade surda, a desassistência inviabiliza o acesso da pessoa com surdez aos recursos básicos de saúde, dificultando a promoção e prevenção dos agravos.

Este estudo possibilitou evidenciar que as dificuldades para uma abordagem adequada desse grupo estão relacionadas a falta de profissionais capacitados em atender a essa demanda linguística, desde a abordagem inicial, compreensão dos agravos em saúde, até a conduta e manejo em relação ao paciente. Ademais, foi observado que as problemáticas para o atendimento integral à comunidade surda estão relacionadas diretamente a intermediação comunicativa inadequada entre o profissional e o paciente com surdez e, sobretudo, ao desconhecimento e/ou não utilização da LIBRAS por estes profissionais.

Investimentos em capacitação continuada na língua de sinais faz-se indispensável em todos os níveis de complexidade da atenção. Além de ter o direito de compreender o que estar sendo falado sobre sua situação de saúde, o paciente com surdez deve ter a possibilidade de decidir, assim como o paciente ouvinte, em aderir ou não a terapêutica proposta, se posicionar durante 0 atendimento e, também, praticar o autocuidado.

Portanto, o conhecimento e a prática da LIBRAS nos locais de atenção à saúde garante a compreensão mútua, a abordagem integral, a escuta qualificada e, principalmente, a superação da barreira comunicativa que tanto afasta os profissionais da saúde do paciente surdo.

"Ao longo da minha formação pedagógica tive a oportunidade de construir, desconstruir e refletir sobre diversos temas ambientais. Entender a importância dos recursos naturais e interferências/transformações antrópicas do espaço geográfico" (Participante 02).

"Conheço algumas causas e consequências dos problemas ambientais e alternativas para amenizálos. Porém, como toda educadora, estudiosa e 
pesquisadora, necessito estar me atualizando em relação a esta temática, repensando antigas práticas e descobrindo novos caminhos na busca da sustentabilidade ambiental" (Participante 06).

"A educação ambiental é acrescentar, sensibilizar o público em geral sobre a importância da questão ambiental, que está intrinsecamente ligada à realidade do nosso dia a dia, é uma questão ou tema interdisciplinar que envolve questões não somente ao nosso entorno, mas temas globais. É necessário contextualizar, explicar os fatos e consequências sobre conservação, sustentabilidade como foco na realidade socioeconômica. Aquele que pratica a educação ambiental no âmbito de ensino é conhecido como "educador ambiental" e não precisa, necessariamente, ser um professor. Pois trata-se, como foi citado, de uma área interdisciplinar que envolve toda a comunidade da sociedade. Quem está voltado à área possui ações e atitudes ou competências voltadas para a conservação do meio ambiente, pode de alguma forma multiplicar conhecimentos e ações essenciais à qualidade de vida e sustentabilidade" (Participante 07).

"Em mim existe essa consciência e a necessidade de conscientizar outras pessoas, levando em consideração a minha formação acadêmica, intelectual e contexto social. Me sinto apta a trabalhar como educador na área, e ressaltando ainda a minha vontade de aprender cada vez mais no âmbito da educação ambiental" (Participante 09).

Grande parte das perguntas tinha uma opção, sendo que as questões relacionadas às ações, recursos e problemas ambientais foram de múltipla escolha, já que os sujeitos participantes poderiam estar envolvidos em diversas atividades, bem como apresentar uma série de aspectos que necessitam de discussão, pois o tema "sustentabilidade" é muito amplo e leva os pesquisadores à investigação e questionamentos para si mesmos e para outros indivíduos, no que diz respeito às mais diversas situações com enfoque na Educação Ambiental, para este contexto. Somente a última pergunta concedeu uma abertura maior, para que os participantes refletissem mais sobre a sua formação, olhando "para dentro de si", a fim de descobrirem e opinarem se realmente estão prontos para atuarem na qualidade de educadores ambientais, ainda que não seja diretamente em uma sala de aula.

Alencar e Barbosa 6 afirmam que "o Brasil pode ser visto como um dos países com um maior conjunto normativo de proteção ambiental, no entanto, na maioria dos casos, o problema esbarra na falta de suporte e de conhecimento necessário sobre tais questões" (p. 232). Isso leva a refletir que, de fato, a educação é a chave para as mudanças ambientais, e que temas como o deste estudo são fundamentais para a formação acadêmica, bem como para o incentivo à adoção de estratégias de sustentabilidade na sala de aula, no ambiente de trabalho, enfim, no contexto em que os formadores de opinião estão inseridos, não na perspectiva de apontar, mas de incentivar uma nova visão de mundo, que traga esperança e a possibilidade de reconstrução da natureza, a partir da preservação dos recursos que ainda se encontram disponíveis, para esta e as futuras gerações.

Os temas destacados para discussão, pelos participantes, refletem 0 quanto 0 ser humano se corrompeu diante dos processos da globalização, o que o levou a conviver e a viver de forma inconsequente e irresponsável, pois grande parte dos desastres que hoje são contemplados são frutos das ações antrópicas. Os recursos têm sido explorados de forma demasiada, sem uma visão consciente, que tem conduzido à escassez como também para a extinção das diversas formas de vida. Por outro lado, há muitos que estão insensíveis, por não terem presenciado, "sentido na pele" uma situação de desastre ou desgaste ambiental, mesmo os noticiários alertando e apresentando cotidianamente como o país se encontra: em meio a uma crise socioambiental que pode ser revertida. Não obstante, a educação no ensino superior ainda precisa de reflexões sólidas em suas propostas curriculares que, de alguma forma, gerem mudanças racionais, práticas pedagógicas e didáticas, e compreensão profunda da problemática ambiental, capazes de sistematizar, divulgar e propor experiências em sustentabilidade ${ }^{10,11}$.

Zupelari e Wick $^{12}$ refletem que a humanidade se encontra diante de uma incerteza em relação ao futuro, pois há um desconforto sendo gerado pela degradação dos recursos, aliado às pressões do capitalismo, ao desbalanço das relações sociais, estas que estão repletas de angústia e insegurança. Os participantes enfatizaram a importância do lúdico, bem como de ações educacionais em saúde que possam reverter esse quadro, por meio de estratégias que despertem a atenção para uma realidade e convoquem o povo para uma mudança que já se encontra em estado de urgência. Estratégias educacionais, na visão de Jesus ${ }^{13}$, intercalam saberes e recursos que podem alcançar as famílias e comunidades, de modo a ultrapassar a dimensão biológica, para então abrir espaço para uma discussão que envolve a qualidade e as dimensões cotidianas, alicerçando o conhecimento científico ao popular, em adaptação para cada contexto, a favor do cumprimento do direito à saúde e qualidade de vida proposto constitucionalmente.

Essas atividades podem ser realizadas não somente nas universidades, mas nas escolas, que são como uma porta de entrada para a inserção das competências, atitudes, valores e habilidades essenciais para a promoção da qualidade de vida e manutenção do meio ambiente. A música, o teatro, as palestras com profissionais inseridos no contexto, as visitas às instituições competentes, são algumas das atividades que os pesquisadores, os acadêmicos e profissionais podem desenvolver para todos os níveis de ensino, com ênfase na Educação Básica, a partir de ações concretas. Sendo que a sensibilidade individual é tarefa da escola e de toda a sociedade, devendo todos os cidadãos estar cientes dos seus direitos e deveres quanto ao meio ambiente, e das inter-relações existentes, o que demanda a adoção de um programa eficiente em prol da preservação dos recursos naturais ${ }^{14,15}$.

Por outro lado, a preocupação com a sustentabilidade ambiental já é uma evidência para a sociedade, apesar de 
que há um longo caminho a ser percorrido. Os municípios têm criado leis que regulamentam, por exemplo, a coleta seletiva, mas cabe a cada cidadão 0 respeito e cumprimento dessas exigências, o que também é um dever das empresas diante da sociedade. Os sujeitos do estudo demonstraram essa preocupação e têm agido por meio de diversas atividades, com destaque à divulgação de causas ecológicas. Alguns realizam atividades prático-ecológicas, como plantio de árvores, separação do lixo para reciclagem e, cotidianamente, eles economizam água e energia elétrica, em sua maioria, o que pode ser considerado como um grande diferencial. Vale lembrar que o "meio ambiente" encontra-se entre os temas transversais e interdisciplinares, no que diz respeito ao currículo escolar brasileiro (inserido nos Parâmetros Curriculares Nacionais), e deve ser promovido por meio do vínculo entre as disciplinas, integrando as áreas do conhecimento, alicerçando ciência, trabalho, arte e tecnologia, bem como repercutir sobre os diferentes setores sociais, como educação, saúde, alimentação, agricultura, transportes e outros $^{15,16}$.

O questionário finaliza com a necessidade de se refletir sobre o que é ser, de fato, um educador ambiental, e se os pós-graduandos estão preparados para esta tarefa que não é nada fácil. Nesse aspecto, é preciso ter conhecimento sobre as tendências pedagógicas e, de algum modo, alicerçá-las à sua própria formação, ainda que o curso de graduação não tenha promovido essa relação, o que se observa amplamente nas formações para o bacharelado, que não possui ligação direta com a atuação para a docência. No entanto, é certo que a universidade funciona como centro investigativo, devendo os profissionais que são educadores ambientais compreender, refletir e analisar os processos aqui apresentados sob uma perspectiva ambiental, bem como as diversas políticas relacionadas, visto que a sua formação está direcionada para a sustentabilidade. Os cidadãos devem ser orientados, a partir dos espaços de educação, o que começa pela qualificação do educador, para então consolidar a sua prática e, portanto, ampliar a sua discussão sobre a natureza e sociedade, em comunhão com as diversas áreas do conhecimento ${ }^{17}$.

\section{CONSIDERAÇÕES FINAIS}

Ter uma visão voltada para a sustentabilidade permite ao cidadão agir no presente, conscientizando a si mesmo e à comunidade em que está inserido, satisfazendo-se pelo uso do que o ambiente dispõe, de forma a não prejudicar as futuras gerações. Os profissionais participantes apresentaram este perfil, esta preocupação, e deixaram claro que os conceitos da Educação Ambiental já fazem parte de sua formação, desde as primeiras construções no âmbito universitário, apesar de alguns ainda não se sentirem preparados para atuar nesta modalidade como educadores. Salientando que atuar na Educação Ambiental não demanda ser um licenciado nem ter uma formação voltada para a sala de aula, mas estar qualificado para abordar e trabalhar com as questões relacionadas ao meio ambiente, o que implica ensino, pesquisa e extensão em quaisquer níveis, mediante a sua dedicação. No entanto, torna-se clara a dimensão do problema, como também o interesse de diversas áreas de atuação em refletir sobre os aspectos apresentados neste texto, colaborar em suas próprias ações cotidianas e imprimir esses aspectos na atuação acadêmica.

\section{CONFLITOS DE INTERESSES}

Não existem conflitos de interesses no que diz respeito à submissão / publicação deste manuscrito em periódicos científicos.

\section{REFERÊNCIAS}

1. Piccoli AS, Kligerman DC, Cohen SC, Assumpção RF. A Educação Ambiental como estratégia de mobilização social para o enfrentamento da escassez da água. Ciênc. Saúde Colet. 2016; 21(3):797-808.

2. Brasil. Presidência da República. Casa Civil. Subchefia para Assuntos Jurídicos. Lei no. 9.795, de 27 de abril de 1999. Dispõe sobre a educação ambiental, institui a Política Nacional de Educação Ambiental e dá outras providências. Brasília, DF; 1999.

3. Brasil. Ministério das Cidades. Secretaria Nacional de Saneamento Ambiental. Programa de Educação Ambiental e Mobilização Social em Saneamento. Caderno metodológico para ações de educação ambiental e mobilização social em saneamento. Brasília, DF; 2009.

4. Barboza LAS, Brasil DSB, Conceição GS. Percepção ambiental dos alunos do $6^{\circ}$ e do $9^{\circ}$ anos de uma escola pública municipal de Redenção, Estado do Pará, Brasil. Rev. Pan-Amaz Saúde. 2016; 7(4):11-20.

5. Gouveia N. Saúde e meio ambiente nas cidades: os desafios da saúde ambiental. Saude Soc. 1999; 8(1):49-61.

6. Alencar LD, Barbosa MFN. Educação Ambiental no Ensino Superior ditames da Política Nacional de Educação Ambiental. RDAS. 2018; 8(2):229-55.

7. Marconi MA, Lakatos EM. Fundamentos de metodologia científica. 6.ed. São Paulo, SP: Atlas; 2005.

8. Prodanov CC, Freitas EC. Metodologia do trabalho científico: métodos e técnicas da pesquisa e do trabalho acadêmico. 2.ed. Novo Hamburgo: Feevale; 2013

9. Online Pesquisa. Criar Pesquisa. Zurich, Switzerland, 2019. [acesso em 2019 jun 15] Disponível em: https://www.onlinepesquisa.com/criarpesquisa.

10. Leff E. Educação Ambiental e Desenvolvimento Sustentável. In: Reigota M. (org.) Verde cotidiano, o meio ambiente em discussão. Rio de Janeiro: $D P \& A ; 1999$

11. Silva ML. A educação ambiental no ensino superior brasileiro: do panorama nacional às concepções de alunos (as) de pedagogia na Amazônia. Revista Eletrônica do Mestrado em Educação Ambiental. 2013; esp.18-33.

12. Zupelari MFZ, Wick MAL. A incerteza do futuro e a questão ambiental na contemporaneidade. Rev. Subj. 2015; 15(1):9-16.

13. Jesus SJA. O papel da educação em saúde frente às implicações da Atenção Básica: do profissional á comunidade. Revista Interfaces: Saúde, Humanas e Tecnologia. 2015; 2(7):1-9.

14. Tramontina LT, Carniatto I. Influências da Educação Ambiental, do grau de escolaridade e do ambiente de trabalho em práticas ambientais por trabalhadores na indústria. Revbea. 2019; 14(1):29-48.

15. Paixão JA. Educação ambiental na Educação Básica: elementos para se pensar o trabalho da dimensão ambiental nas aulas de Educação Física. Horizontes. 2018; 36(1):196-207.

16. Brasil. Secretaria de Educação Fundamental. Parâmetros Curriculares Nacionais: Meio Ambiente. Brasília, DF; 1997.

17. Morales AGM. A formação dos profissionais educadores ambientais e a universidade: trajetórias dos cursos de especialização no contexto brasileiro. Educar, Curitiba. 2009. (34):185-99. 